\title{
Treatment of myoclonic seizures in patients with juvenile myoclonic epilepsy
}

\author{
Stéphane Auvin \\ Department of Pediatric Neurology, \\ Lille University Hospital, Lille, France; \\ Pharmacology Laboratory, Lille \\ Medical School, Lille, France
}

\begin{abstract}
Drug treatment of Juvenile myoclonic epilepsy (JME) is mainly based on clinical experience and prospective and retrospective studies, with little evidence from randomized clinical trials. There are no head-to-head comparisons between old and new antiepileptic drugs (AEDs) and no drugs licensed specifically for JME. Valproate is unquestionably the drug of the first choice in men with JME. In women, lamotrigine should be preferred regarding teratogenicity and side effects of valproate. In addition, levetiracetam and topiramate are effective and can be use in combination or as second line treatment. Some AEDs can aggravate JME. In addition of AEDs, non-pharmacological treatments are important in JME. JME usually require lifelong treatment because seizures nearly always return after withdrawal of therapy.
\end{abstract}

Keywords: myoclonic seizure, myoclonic epilepsy, antiepileptic drugs

\section{Introduction}

Juvenile myoclonic epilepsy (JME) is a common epilepsy syndrome that is classified among the idiopathic generalized epilepsies (IGEs) (ILAE 1989). It is a collection of seizure patterns that are clinically distinct from those in other forms of IGE, with the major characteristic being adolescence-onset myoclonic seizures. Although the pathogenesis is unknown, recent advances, especially in genetics, suggest several possible mechanisms. JME is both genetically and clinically heterogeneous; some clinical differences may indicate differences in pathogenesis in different groups of patients. The nosology and classification of IGE syndromes are currently under revision (Andermann and Berkovic 2001; Engel 2001). JME is characterized by a particular pharmacological sensitivity. Valproate has a specific potency. Others antiepileptic drugs (AEDs) are also efficient, such as lamotrigine, clonazepam, topiramate and levetiracetam, while others may have aggravating effects.

\section{Electroclinical features of juvenile myoclonic epilepsy}

JME is a common IGE that is characterized by myoclonic seizures. It is the most common IGE in adults, especially in women. Isolated myoclonic jerks of the arms, especially shortly after awakening, are characteristic. Generalized tonic-clonic seizures often occur, and one-third of individuals have absences. The generalized tonic-clonic seizures are often preceded by a few minutes of generalized myoclonus of increasing frequency and intensity. They occur predominantly after awakening. Seizure occurrence is more likely with sleep deprivation, fatigue and alcohol withdrawal. Onset is usually in adolescence but seizures may begin or be diagnosed only in the early 20 s. Eighty percent of patients with JME begin having seizures between ages 12 and 18 . The mean age of onset for generalized tonic clonic seizures is 15.5 -years. The most important element in the diagnosis of JME is the clinical history.

The typical abnormality on electroencephalography (EEG) is bilateral multiple spike or polyspike-wave complexes which precede a myoclonic jerk. The
Correspondence: Stéphane Auvin

Pediatric Neurology Department, Hôpital Roger Salengro, 59037 Lille Cedex, France Tel +33 320444057

Fax +33 20445393

Email auvin@invivo.edu 
EEG discharge includes 5-20 spikes with a frequency between 12 and $16 \mathrm{~Hz}$. The amplitude of spikes is frequently maximal in anterior areas. An ictal recording is best obtained by polygraphic video-EEG performed a provoked early morning awakening during a full-night sleep recording or at least after noctural sleep deprivation.

$\mathrm{JME}$ is one of the epileptic syndrome clearly associated with photosensitivity occurring in about 30\% (Janz 1985; Loiseau and Duche 1990). Myoclonic seizure can be induced by intermittent luminous stimulation in photosensitive patients, but clinical photosensitivity is less frequent than during EEG recording.

In some cases, focal clinical or EEG features or both in patients with JME can be observed. These features include not only unilateral myoclonic jerks but also version and asymmetrical tonic limb posturing (Aliberti et al 1994; Lancman et al 1994; Usui et al 2005).

Misdiagnosis and delayed diagnosis remain common, as reported in the literature (Grunewald et al 1992; Atakli et al 1998). Patients frequently come to medical attention only after a generalized convulsion, and the history of much earlier myoclonic jerks is then often obtained only retrospectively (Grunewald et al 1992).

\section{Treatment}

The goal of management is to render the individual seizurefree without the side effect of the medication. JME require lifelong treatment because seizures nearly always return after withdrawal of therapy (Delgado-Escueta and Enrile-Bascal 1984; Baruzzi et al 1998). JME is difficult to treat in about $15 \%$ of patients. The predictors of pharmacoresistance include: (1) the coexistence of all three seizures types (myoclonic jerks, absence seizures and GTCS), (2) the existence of associated psychiatric problems, and (3) focal clinical symptoms and/or focal EEG discharge (Dasheiff and Ritaccio 1993; Fernando-Dongas et al 2000; Gelisse et al 2001).

\section{Non-pharmacological treatment}

In JME, lack of sleep and alcohol intake can induce seizures. The sleep-wake rhythm has to be regulated. The circumstances interfering with normal sleep and progressive awakening in the morning should be eliminated. If the patient stays up late at night, he should sleep longer the next morning. Alcoholic drinks will only permitted in small quantities, on social occasions. Patients should be advised not to drink in cases of lack of sleep. If the patient has photosensitivity, they should avoid relevant visual stimuli. Some maneuvers reduce the risk of photosensitive seizures, including monocul complete occlusion, increased distance from a video-display terminal, and the provision of ambient back lighting (Harding and Jeavons 1992). We suggest these approaches to patients who show photosensitivity on EEG but do not know how successful these environmental alterations are in JME.

\section{Valproate}

\section{Mechanism of action}

Valproic acid is a branched-chain fatty acid that markedly differs in structure from all other AEDS in clinical use. Its mechanism of action remains not fully understand. There are evidences that valproic acid increases GABA concentrations, the mechanism by which this occurs remains unknown. A number of mechanisms have been proposed, including inhibition of GABA-T action, activation of GABA synthesis by an increase of GAD activity, and increased release of GABA into the synapse.

\section{Efficacy}

Based on clinical experience, valproate was first proposed as a treatment of myoclonic seizure. 22-years ago, the sensitivity of VPA was reported (Delgado-Escueta and Enrile-Bascal 1984). Although JME is an important syndrome, there have been almost no randomized clinical trials conducted using any medication for its treatment. However, there have been many open case studies conducted with VPA in JME management. The open series that have been published using VPA show a $41 \%-88 \%$ seizure-free rate for patients receiving VPA, either as an add-on medication or as monotherapy (Atakli et al 1998; Kleveland and Engelsen 1998). Case studies have also shown that a low, once-daily dose $(500 \mathrm{mg})$ of VPA can effectively control JME and keep patients seizure free for as long as 2-years (Panagariya et al 2001; Karlovassitou-Koriari et al 2002). Newer studies that examined the efficacy of lamotrigine, topiramate and valproate in JME have all shown that valproate has the best efficacy of the three (Prasad et al 2003; Nicolson et al 2004; Mohanraj and Brodie 2005).

Valproate is today one of the most effective AEDs in JME. He should be considered the first line drug at least for man. Taking into an account the teratogenicity (possibly lessened by the concurrent use of folic acid) and some side effects (weight gain), VPA are often considered unacceptable in young women of childbearing age.

\section{Benzodiazepin}

Mechanism of action

The benzodiazepines work by primarily enhancing gammaaminobutyric acid (GABA)ergic inhibition by binding to the benzodiazepine receptor on GABAA receptors (Rudolph et al 1999). 


\section{Efficacy}

Clonazepam can also be effective in the treatment of JME. Obeid and Panayiotopoulos (1989) treated 17 patients who had JME with Clonazepam. All of the patients had myoclonic seizures before treatment, and 14 of the 17 had GTC seizures before treatment. After Clonazepam treatment, myoclonic seizures were controlled in $15(88 \%)$ of 17 patients, and the remaining two patients had a $75 \%$ reduction in seizure frequency. However, Clonazepam did not produce effective results for reducing GTC seizures. Six (43\%) of the 14 patients with GTC seizures were controlled. In addition, two patients sustained injuries during GTC seizures because Clonazepam eliminated their myoclonic seizures, which had served as a warning, and they went directly into a GTC seizure. The authors concluded that Clonazepam, while effective in treating myoclonic seizures, probably should not be used to control GTC seizures.

\section{Lamotrigine}

\section{Mechanism of action}

Lamotrigine is a phenyltriazine derivative. Lamotrigine acts through inhibition of voltage-activated sodium channels and possibly calcium channels, so that it prevents the release of glutamate (Lees and Leach 1993).

\section{Efficacy}

Lamotrigine is effective in controlling generalized tonic clonic seizure and absence while there are some reports of myoclonic seizures exacerbation (Biraben et al 2000; Carrazana and Wheeler 2001).

Many studies performed over recent years have demonstrated clinical utility for the treatment of JME. An Open-label study to evaluate lamotrigine as an alternative to valproate (63 patients switched from valproate to lamotrigine) demonstrated an improvement in $67 \%$ of patients. No aggravation of clinical symptoms was also reported (Morris et al 2004). Buchanan conducted a study of 12 patients with JME who were treated with LTG monotherapy (Buchanan 1996). Five (41\%) of these 12 patients became seizure free while receiving LTG monotherapy, and the authors concluded that LTG might be effective in the management of JME. In a retrospective study, valproate monotherapy $(n=36)$ and lamotrigine monotherapy $(\mathrm{n}=14)$ were compared, as well valproate $(n=22)$, lamotrigine $(n=21)$ and topiramate polytherapy $(\mathrm{n}=15)$ were compared. The results suggested that lamotrigine were an effective option as monotherapy and polytherapy (Prasad et al 2003). Furthermore, the efficacy of lamotrigine in JME was evaluated in bitherapy.
The polytherapy is especially interesting in case of drug resistance. The combination of lamotrigine with valproate appears to be synergistic in many type of epilepsy. But the same combination is lead to an increased risk to develop an important skin rash (Gerike et al 1999; Pisani et al 1999).

\section{Levetiracetam}

\section{Mechanism of action}

Levetiracetam is a pyrrolidone derivative, is the S-enantiomer of the ethyl analogue of piracetam. While the exact mechanism of action of levetiracetam is not known, it appears to act via a specific binding site within the brain (but does not directly affect GABA or glutamate), does not alter sodium channel properties, produces a limited reduction in highvoltage-activated calcium currents, and possibly modulates intracellular calcium transients (Herdon et al 1997; Zona et al 2001). Molecular studies involving transgenic mice suggest that levetiracetam binds to a synaptic vesicle protein (SVA2) that is involved in vesicle neurotransmitter exocytosis, and that the binding affinity to SVA2 is directly proportional to seizure protection (Lynch et al 2004).

\section{Efficacy}

Levetiracetam is probably the best new AED in the treatment of JME and may replace valproate for the treatment of the disorder because of high and sustained efficacy, fast action, and an excellent safety profile. More than $60 \%$ of patients with intractable JME became seizure-free with levetiracetam monotherapy or polytherapy (Czapinski and Czapinska 2004; Resor and Resor 2005). Resor and colleagues assessed levetiracetam monotherapy in 24 patients with JME and GTCS (Resor and Resor 2005). Sixteen patients $(66.7 \%)$ became free of GTCS; three of them had a single convulsive seizure after either stopping levetiracetam for $24 \mathrm{~h}$ or reducing the dose to $500 \mathrm{mg} /$ day but subsequently have remained free of convulsive seizures. Myoclonic seizures were effectively controlled in 22 of 24 patients. Moreover, Sharpe and colleagues performed a retrospective review on the medical record of 30 patients with JME, whose age was between 5 and 21 -years, treated with levetiracetam with dosage range from 12 to $50 \mathrm{mg} / \mathrm{kg} / \mathrm{j}$ (Sharpe et al 2005). The length of follow-up was 27 -months. 24 patients $(80 \%)$ became seizure free with levetiracetam monotherapy and 2 additional patients had an improvement of seizure control. Andermann and colleagues evaluated levetiracetam in 122 patients (113 with JME and 9 with juvenile absence epilepsy). The study was a double-bind multicenter placebo controlled trial conducted in patients 
without control on one AED. The responder rate was 58.3\% with levetiracetam versus $23.3 \%$ with placebo (Andermann et al 2005). Specchio et al conducted a multicenter, prospective open-label study in patients with JME (Specchio et al 2006). 10 patients were newly diagnosed and 38 were resistant and/or intolerant to previous AED. 5 patients dropped out. 18 patients $(37.5 \%)$ were without myoclonia and 35 (73\%) had no generalized tonic seizure.

\section{Topiramate}

\section{Mechanism of action}

Topiramate is a sulphamate-substituted monosaccharide that exhibits some inhibition of carbonic anhydrase. Its AED activity is independent of this effect. Topiramate has multiple modes of action, including modulation of voltage-dependent sodium channels, potentiation of GABAergic inhibition at a novel site on the GABAA receptor, and possible action at non-Nmethyl- D-aspartate (NMDA) receptors (Hanaya et al 1998; White et al 2000; Zona et al 1997).

\section{Efficacy}

Several studies have shown the usefulness of topiramate in patients with JME. There is one randomized-controlled study on the efficacy of topiramate in patients with generalized tonic clonic seizure including patients with JME (Biton et al 2005). A reduction of more than $50 \%$ of generalized tonic clonic seizure were reported in $8 / 11$ patients treated by topiramate compare to $2 / 11$ in placebo-treated patients. A reduction in myoclonic and absence seizures was also reported but did not achieve statistical significance. Topiramate efficacy and tolerability were evaluated in 22 patients with JME (Sousa Pda et al 2005). 16 patients completed the first year of the follow-up, while 4 left the study because of poor seizure control and 2 because of low compliance. Myoclonic seizures were controlled in 11 patients and persisted in 5 patients. Generalized tonic clonic seizures were completely controlled in 10 patients, 4 patients had more than $50 \%$ reduction of seizures and 2 had reduction less than $50 \%$. In a randomized open-label treatment, 28 patients with a recent diagnosed JME received topiramate or valproate (Levisohn et al 2003). 12 of 19 patients treated by topiramte completed the study, while 7 of 9 patients treated by valproate completed the study. Generalized tonic clonic, myoclonic and absence seizures were totally controlled in 10/12, 9/14 and $2 / 2$ topiramate-treated patients, respectively, and 3/4, 7/9 and $1 / 2$ valproate-treated patients. In a retrospective cohort, topiramate monotherapy and polytherapy were considered as an alternative to VPA (Prasad et al 2003).

\section{Zonisamide}

Mechanism of action

Zonisamide by blocking the voltage-sensitive sodium channels prevents repetitive neuronal firing. It also reduces voltage-dependent T-type calcium channels, facilitates dopaminergic and serotoninerigic neurotransmission, weakly inhibits carbonic anhydrase and may protect neurones from free-radical damage, thereby stabilizing neuronal membranes (Suzuki et al 1992; Kito et al 1996).

\section{Efficacy}

Few studies focused on the effects of zonisamide are available. A retrospective study analyzing 15 patients (monotherapy in 13 patients and added to valproate in 2 patients) reported a good control of seizures. $69 \%, 62 \%$, and $38 \%$ were free for generalized tonic clonic, myoclonic and absence seizures, respectively. Side effects were reported in $20 \%$ of patients (Kothare et al 2004). In a neurophysiological study of a patient, zonisamide permits almost complete disappearance of generalized spike and wave discharges (Szaflarski 2004). These data suggest the possible useful of this AED in JME.

\section{AED aggravating JME}

It is now well known that AEDs can aggravate epilepsy. Phenytoin and carbamazepine have shown to aggravate the myoclonic and absence seizures of JME and therefore should be avoided (Genton et al 2000; Gelisse et al 2004; Thomas et al 2006). Genton et al (2000) conducted a study with $\mathrm{CBZ}$ and PHT to determine the risk of seizure aggravation in 40 patients with JME. In the CBZ group, $19(68 \%)$ of 28 patients had a seizure aggravation, usually affecting the myoclonic seizure, and only six (21\%) of 28 patients improved. In the PHT group, six (38\%) of 16 patients had a seizure aggravation, and only two (12\%) of 16 patients improved. Aggravation mostly was in the form of increased myoclonic jerks. The authors concluded that among commonly prescribed AEDs, CBZ appears to have the strongest aggravating potential in patients with JME, whereas the aggravating effect of PHT is less prominent.

There have been isolated series of reports indicating that lamotrigine can exacerbate or aggravate myoclonic seizures. Biraben et al reported four patients with JME receiving $\mathrm{LTG}$ therapy that experienced myoclonus seizure aggravation (Biraben et al 2000). Carrazana et al also found two patients with JME (2/24) developed dramatic exacerbation of myoclonus and two other $(2 / 24)$ had mild transient 
increases in morning myoclonus with lamotrigine (Carrazana and Wheeler 2001).

\section{Antiepileptic drug selection}

When presented with a patient with epilepsy, physicians should prescribe the medication that will effectively control the seizures yet not endanger the patient with adverse events or seizure aggravation. Although JME is an important syndrome, there have been no randomized clinical trials conducted using any medication for its treatment. Drug treatment of JME is mainly based on clinical experience and prospective and retrospective studies. There are no head-to-head comparisons between old and new AEDs and no drugs licensed specifically for JME.

The 2001 and 2005 expert consensus surveys on adult epilepsies (Karceski et al 2001, 2005) ranked valproate as the treatment of choice for idiopathic generalized epilepsy when the patient's sex was not specified. However, in the 2005 survey adult epilepsy, when it was specified that the patient was a woman, the experts rated lamotrigine as treatment of choice. In the 2005 expert opinion about pediatric epilepsy treatment, valproate and lamotrigine was the for 15-year-old male with JME, while lamotrigine was the only choice for 15-year-old female with JME (Wheless et al 2005).

Valproate is unquestionably the drug of the first choice in men with JME. In women, where considerations of teratogenicity, weight gain, and polycystic ovary syndrome are relevant, other options include levetiracetam, lamotrigine, topiramate and perhaps zonisamide. In patients with symptoms refractory to treatment with one drug, valproate with small doses of lamotrigine is often an effective combination. When valproate is undesirable, combining levetiracetam with lamotrigine may be effective.

\section{References}

Aliberti V, Grunewald RA, Panayiotopoulos CP, et al. 1994. Focal electroencephalographic abnormalities in juvenile myoclonic epilepsy. Epilepsia, 35:297-301.

Andermann E, Andermann F, Meyvisch P, et al. 2005. Seizure control with levetiracetam in juvenile myoclonic epilepsies. Epilepsia, 46(Suppl 8):205.

Andermann F, Berkovic SF. 2001. Idiopathic generalized epilepsy with generalized and other seizures in adolescence. Epilepsia, 42:317-20.

Atakli D, Sozuer D, Atay T, et al. 1998. Misdiagnosis and treatment in juvenile myoclonic epilepsy. Seizure, 7:63-6.

Baruzzi A, Procaccianti G, Tinuper P, et al. 1998. Antiepileptic drug withdrawal in childhood in childhood epilepsies: preliminary results of a prospective study. In: Faienza C, Prati G eds. Diagnostic and therapeutic problems in pediatric epileptology. Amsterdam: Elsevier. p 117-23.

Biraben A, Allain H, Scarabin J, et al. 2000. Exacerbation of juvenile myoclonic epilepsy with lamotrigine. Neurology, 55:1758.

Biton V, Bourgeois B. YTC/YTCE study investigators. 2005. Topiramate in patients with juvenile myoclonic epilepsy. Arch Neurol, 62:1705-8.
Buchanan N. 1996. The use of lamotrigine in juvenile myoclonic epilepsy. Seizure, 5:149-51.

Carrazana E, Wheeler S. 2001. Exacerbation of juvenile myoclonic epilepsy with lamotrigine. Neurology, 56:1424-5.

Czapinski PP, Czapinska EM. 2004. The effectiveness of levetiracetam in drug-resistant juvenile myoclonic epilepsy. Epilepsia, 45(Suppl 3):141.

Dasheiff RM, Ritaccio AL. 1993. Characterization of intractable juvenile myoclonic epilepsy: new perspectives on primarily generalized seizures. Seizure, 2:11-19.

Delgado-Escueta A, Enrile-Bascal F. 1984. Juvenile myoclonic epilepsy of Janz. Neurology, 34:285-94.

Engel J. 2001. A proposed diagnostic scheme for people with epileptic seizures and with epilepsy: report of the ILAE Task Force on Classification and terminology. Epilepsia, 42:1-8.

Fernando-Dongas MC, Radtke RA, VanLandingham KE, et al. 2000. Characteristics of valproic acid resistant juvenile myoclonic epilepsy. Seizure, 9:385-8.

Gelisse P, Genton P, Kuate C, et al. 2004. Worsening of seizures by oxcarbazepine in juvenile idiopathic generalized epilepsies. Epilepsia, 45:1282-6.

Gelisse P, Genton P, Thomas P, et al. 2001. Clinical factors of drug resistance in juvenile myoclonic epilepsy. J Neurol Neurosurg Psychiatry, 70:240-3.

Genton P, Gelisse P, Thomas P, et al. 2000. Do carbamazepine and phenytoin aggravate juvenile myoclonic epilepsy? Neurology, 55:1106-9.

Gerike C, Picard F, Saint-Martin A, et al. 1999. Efficacy of lamotrigine in idiopathic generalized epilepsy syndromes: a video-EEG-controlled open study. Epileptic Disord, 1:159-65.

Grunewald RA, Chroni E, Panayiotopoulos CP. 1992. Delayed diagnosis of juvenile myoclonic epilepsy. J Neurol Neurosurg Psychiatry, 55:497-9.

Hanaya R, Sasa M, Ujihara H, et al. 1998. Suppression by topiramate of epileptiform burst discharges in hippocampal CA3 neurons of spontaneously epileptic rat in vitro. Brain Res, 789:274-82.

Harding W, Jeavons J. 1992. Photosensitive epilepsy. In: Harding W, Jeavons J eds. London: MacKeith Press. p 125-50.

Herdon HJ, Jerman JC, Stean TO, et al. 1997. Characterization of the binding of [3H]-SB-204269, a radiolabelled form of the new anticonvulsant SB-204269, to a novel binding site in rat brain membranes. $\mathrm{Br}$ J Pharmacol, 121:1687-91.

ILAE. 1989. Proposal for revised classification of epilepsies and epileptic syndromes. Commission on Classification and Terminology of the International League Against Epilepsy. Epilepsia, 30:389-99.

Janz D. 1985. Epilepsy with impulsive petit mal (juvenile myoclonic epilepsy). Acta Neurol Scand, 72:449-59.

Karceski S, Morrell M, Carpenter D. 2001. The expert consensus guideline series: Treatment of epilepsy. Epilepsy Behav, 2:A1-50.

Karceski S, Morrell M, Carpenter D. 2005. Treatment of epilepsy in adults: expert opinion. Epilepsy Behav, 7:S1-64.

Karlovassitou-Koriari A, Alexiou D, Angelopoulos P, et al. 2002. Low dose sodium valproate in the treatment of juvenile myoclonic epilepsy. J Neurol, 249:396-9.

Kito M, Maehara M, Wanatabe K. 1996. Mechanisms of T-type channel blockade by zonisamide. Seizure, 5:115-9.

Kleveland G, Engelsen B. 1998. Juvenile myoclonic epilepsy: clinical characteristics, treatment and prognosis in a Norwegian population of patients. Seizure, 7:31-8.

Kothare S, Valencia I, Khurana D, et al. 2004. Efficacy and tolerability of zonisamide in juvenile myoclonic epilepsy. Epileptic Disord, 6:267-70.

Lancman ME, Asconape JJ, Penry JK. 1994. Clinical and EEG asymmetries in juvenile myoclonic epilepsy. Epilepsia, 35:302-6.

Lees G, Leach MJ. 1993. Studies on the mechanism of action of the novel anticonvulsant lamotrigine (Lamictal) using primary neurological cultures from rat cortex. Brain Res, 612:190-9.

Levisohn P, Holland K, Hulihan J, et al. 2003. Topiramate versus valproate in patients with juvenile myoclonic epilepsy. Epilepsia, 44:267-8. 
Loiseau P, Duche B. 1990. Epilepsie myoclonique juvenile. Rev Neurol, 146:719-25.

Lynch BA, Lambeng N, Nocka K, et al. 2004. The synaptic vesicle protein SV2A is the binding site for the antiepileptic drug levetiracetam. Proc Natl Acad Sci USA, 101:9861-6.

Mohanraj R, Brodie M. 2005. Pharmacological outcomes in newly diagnosed epilepsy. Epilepsy Behav, 6:382-7.

Morris G, Hammer A, Kustra R, et al. 2004. lamotrigine for patients with juvenile myoclonic epilepsy following prior treatment with valproate: results of an open-label study. Epilepsy Behav, 5:509-21.

Nicolson A, Appelton R, Chadwick D, et al. 2004. The relationship between treatment with valproate, lamotrigine, and topiramate and the prognosis of idiopathic generalised epilepsies. J Neurol Neurosurg Psychiatry, 75:75-9.

Obeid T, Panayiotopoulos CP. 1989. Clonazepam in juvenile myoclonic epilepsy. Epilepsia, 30:603-6.

Panagariya A, Sureka R, Ardana V. 2001. Juvenile myoclonic epilepsy - an experience from north western India. Acta Neurol Scand, 104:12-16.

Pisani F, Oteri G, Russo M, et al. 1999. The efficacy of valproate-lamotrigine comedication in refractory complex partial seizures: evidence for a pharmacodynamic interaction. Epilepsia, 40:1141-6.

Prasad A, Kuzniecky R, Knowlton R, et al. 2003. Evolving antiepileptic drug treatment in juvenile myoclonic epilepsy. Arch Neurol, 60:1100-5.

Resor SR, Resor LD. 2005. Levetiracetam monotherapy in the treatment of convulsive seizures in juvenile myoclonic epilepsy. Presented at the 54th Annual Meeting of the American Academy of Neurology, April 13-20, 2002; Denver, CO.

Rudolph U, Crestani F, Benke D, et al. 1999. Benzodiazepine actions mediated by specific gamma-aminobutyric acid(A) receptor subtypes. Nature, 401:796-800.
Sharpe DV, Patel A, Fenichel GM, et al. 2005. Use of levetiracetam in juvenile myoclonic epilepsy [abstract]. Epilepsia, 46(Suppl 8):192.

Sousa Pda S, Araujo Fihlo G, Garzon E, et al. 2005. Topiramate for the treatment of juvenile myoclonic epilepsy. Arq Neuropsquiatr, 63:733-7.

Specchio L, Gambardella A, Giallonardo A, et al. 2006. Open label, longterm, pragmatic study on levetiracetam in the treatment of juvenile myclonic epilepsy. Epilepsy Res, 71:32-9.

Suzuki S, Kawakami K, Nishimura S, et al. 1992. Zonisamide blocks T-type channel in cultured neurons of rat cerebral cortex. Epilepsy Res, 12:21-7.

Szaflarski J. 2004. Effects of zonisamide on the electroencephalogram of a patient with juvenile myoclonic epilepsy. Epilepsy Behav, 5:1024-6.

Thomas P, Valton L, Genton P. 2006. Absence and myoclonic status epilepticus precipitated by antiepileptic drugs in idiopathic generalized epilepsy. Brain, 129:1281-92.

Usui N, Kotagal P, Matsumoto R, et al. 2005. Focal semiologic and electroencephalographic features in patients with juvenile myoclonic epilepsy. Epilepsia, 46:1668-76.

Wheless JW, Clarke DF, Carpenter D. 2005. Treatment of pediatric epilepsy: expert opinion. Juvenile myoclonic epilepsy. J Child Neurol, 20:S42-3.

White H, Brown S, Woodhead J, et al. 2000. Topiramate modulates GABAevoked currents in murine cortical neurons by a nonbenzodiazepine mechanism. Epilepsia, 41:17-20.

Zona C, Ciotti MT, Avoli M. 1997. Topiramate attenuates voltagegated sodium currents in rat cerebellar granule cells. Neurosci Lett, 231:123-6.

Zona C, Niespodziany I, Marchetti C, et al. 2001. Levetiracetam does not modulate neuronal voltage-gated $\mathrm{Na}^{+}$and $\mathrm{T}$-type $\mathrm{Ca}_{2}{ }^{+}$currents. Seizure, 10:279-86. 